\title{
Observations On The Ultrastructure Of The Naked Freshwater Flagellate Chrysochromulina tobin sp. nov. (Haptophyta)
}

\author{
Steven B. Barlow ${ }^{1}$ and Rose Ann Cattolico ${ }^{2}$ \\ ${ }^{1}$ Electron Microscope Facility, San Diego State University, San Diego CA USA \\ ${ }^{2}$ Departments of Biology and Ocean Sciences, University of Washington, Seattle WA USA
}

Haptophytes are nano-planktonic unicells whose small size belies their contribution of 30-50\% of the total photosynthetic stock in the world's ocean [1]. In addition to their major role in contributing to the Earth's carbon cycle [1], blooms of these algae can produce noxious odors that compromise water quality and can generate toxins responsible for fish and animal mortality [2]. Because the fatty acids stored by these phytoplankton taxon represent a source of oil, commercial interest in identifying and characterizing Haptophytes that produce large amounts of potential biofuel and high value pharmaceutical products has significantly increased [3].In this report, we present ultra-structural details of a naked freshwater member of the genus Chrysochromulina, most of whose members are characterized by the presence of one or more layers of organic scales covering their surface [4].

Chrysochromulina tobin sp. nov. cell pellets were fixed for TEM 1 hour in $2.0 \%$ glutaraldehyde in 0.15 $\mathrm{M}$ sodium cacodylate buffer ( $\mathrm{pH} 7.2$ ) at room temp., rinsed in buffer followed by $1.0 \%$ osmium tetroxide in $0.15 \mathrm{M}$ sodium cacodylate buffer $(\mathrm{pH} 7.2)$ for 1 hour on ice. Pellets were dehydrated in a graded alcohol series, embedded in EMBed812, and polymerized at $60^{\circ} \mathrm{C}$. Silver sections were cut with a Diatome diamond knife, stained with aqueous uranyl acetate and lead citrate, and viewed on a Tecnai 12 TEM. Images were recorded on an XR-41S 2k digital camera. For SEM, one milliliter of concentrated cell culture was mixed with an equal volume of $4.0 \%$ glutaraldehyde in $0.2 \mathrm{M}$ sodium cacodylate buffer ( $\mathrm{pH} 7.0)$ by inversion for 20 seconds, followed by the immediate addition of 250 microliters of $4.0 \%$ osmium tetroxide. After 20 minutes, cells were collected on 0.2 um Nucleopore filters, dehydrated in an alcohol series, critical point dried, platinum coated, then viewed in a FEG-SEM.

Members of this genus are usually covered with one or more layers of organic scales produced in the Golgi apparatus that are a mainstay of taxonomic identification. Given that C. tobin sp. nov. is an extremely small cell that lacks scales, its taxonomic identity must be based in part on ultrastructural details. Cells are biflagellate, about 5 um in length, either globose or elongated, with a groove that runs the length of the cell (Figure 1). An elongate haptonema, 5x the body length, is either extended or coiled into the groove (Figure 2). No scales are observed either in the Golgi apparatus or on the cell surface. There are two parietal chloroplasts, and mitochondria have tubular cristae (Figure 3). Two lipid bodies are located in the anterior of the cell (Figure 4). In cross-section, the haptonema contains 6 microtubules, with electron dense connections between adjacent tubules. The endoplasmic reticulum extends along the length of the haptonema (Figure 5). Attached to the basal bodies by electron-dense plaques are 4 microtubular rootlets, including one flat ribbon of 9-12 microtubules (R1) (Figure 6). These rootlets extend toward the two parietal chloroplasts. The basal bodies and the haptonema are interconnected by various fibrous roots (Figure 7), some of which appear cross-banded (Figure 8).

The internal morphology of this cell closely resembles other members of this genus, but the lack of scales, coupled with significant differences in genome signature from other recognized Chrysochromulina representatives, supports the delineation of this organism as a new species [5] [6]. 


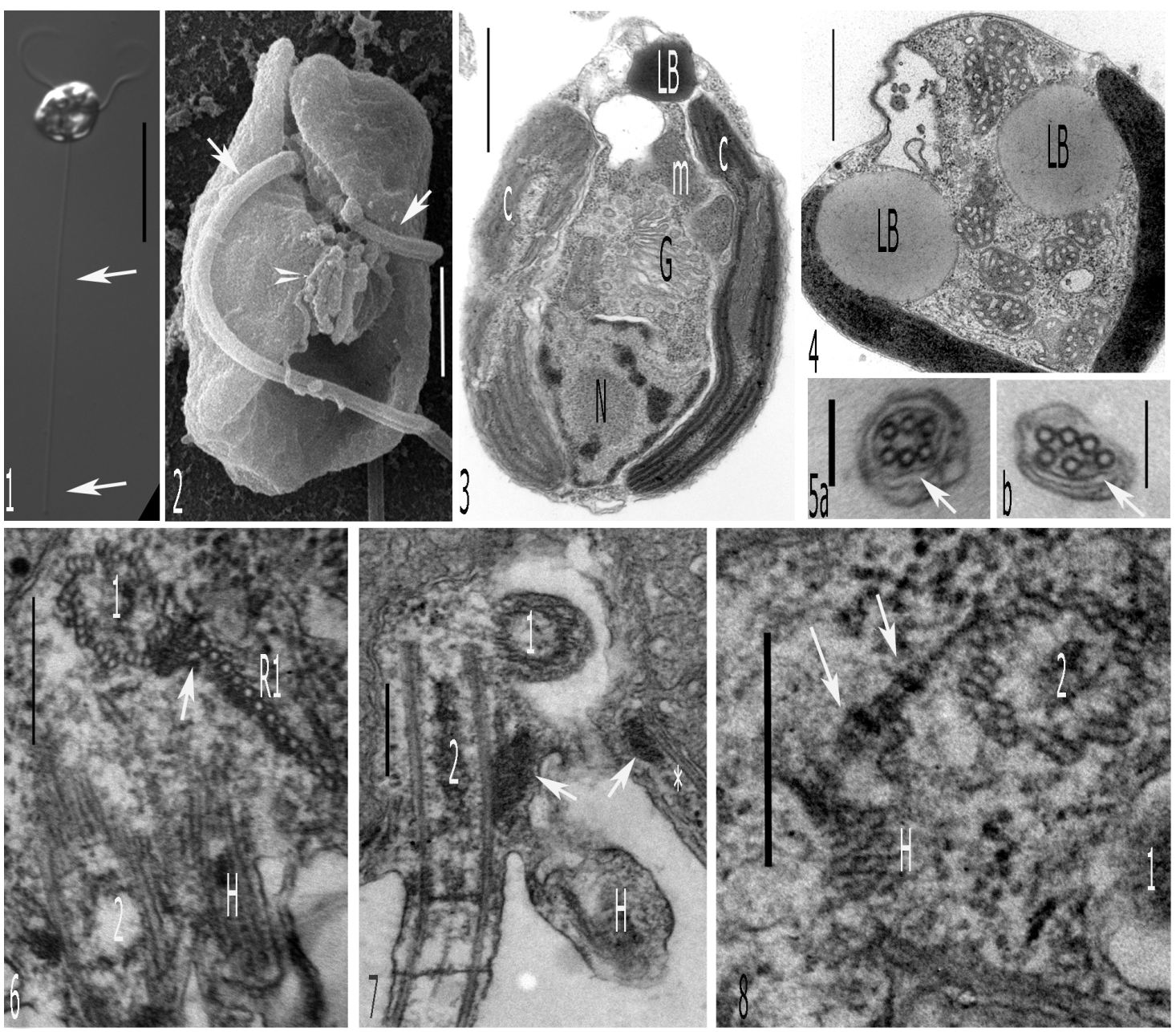

Figure 1. Globose biflagellate cell with elongated haptonema (arrows). Bar=5 um. Figure 2. Longitudinal groove in cell, 2 flagella (arrows) and coiled haptonema (arrowhead). Bar=1 um. Figure 3. Whole cell with lipid body (LB), Golgi apparatus (G), chloroplasts (C), nucleus (N), and mitochondria (M). Bar=1 um. Figure 4. Large lipid bodies (LB) in cytoplasm. Bar=1 um. Figure 5 a, b. Cross-section of haptonema showing 6 crosslinked microtubules and endoplasmic reticulum (arrows). Bar $=250 \mathrm{~nm}$. Figure 6. Microtubular R1 rootlet attached to basal body 1 by e-dense plaque. Bar= $250 \mathrm{~nm}$. Figure 7. Portion of the fibrous rootlet (arrowheads) between basal body 2 and R1 rootlet (asterisk). Bar $=250 \mathrm{~nm}$.

Figure 8. Cross-banded rootlet (arrows) between BB2 and haptonema (H). Bar $=250 \mathrm{~nm}$.

1) H Liu et al, Proc Natl Acad Sci U S A 106 (2009), p. 12803

2) G Hallegraph in "Manual On Harmful Marine Microalgae, G Hallegraph, D Anderson, A Cembella (UNESCO, Paris) p. 1

3) N Bigelow et al, Algal Research (2013) http://dx.doi.org/10.1016/j.algal.2013.07.001

4) B Edvardson et al, Eur J Phycol 46 (2011), p. 202

5) Deodato et al, submitted

6) Supported by NSF instrument grant DBI-030829 to SBB and US Dept. of Energy under Contract DEEE0003046 awarded to the National Alliance for Advanced Biofuels and Bioproducts to RAC. 